\title{
Extensively Drug-Resistant Tuberculosis and Tetralogy of Fallot: Two Demons Together
}

Deepak Rosha ${ }^{1}$, Trilok Chand ${ }^{2 *}$

${ }^{1}$ Senior Consultant, Respiratory, Critical care \& Sleep Medicine, Indraprastha Apollo Hospitals, New Delhi, India

${ }^{2}$ Specialist Pulmonologist, Burjeel Hospital, Abu Dhabi, UAE

\author{
DOI: $10.36348 / \mathrm{sjm} .2020 . \mathrm{v} 05 \mathrm{i} 11.003$ \\ | Received: 04.11.2020 | Accepted: 16.11.2020 | Published: 19.11 .2020 \\ *Corresponding Author: Dr. Trilok Chand
}

\section{Abstract}

The pulmonary tuberculosis is endemic in India, and the incidence of extensively drug-resistant tuberculosis (XDR-TB) is also rising. Though the incidence of pulmonary tuberculosis is high in acyanotic congenital heart disease, in Tetralogy of Fallot, which is a low flow condition, this association is infrequent. We report a rare association of extensively drugresistant tuberculosis and Tetralogy of Fallot in a young adult patient who presented with breathlessness on exertion, hemoptysis and frequent respiratory infections. The patient was successfully managed by bronchial artery embolization, venesections, pulmonary outflow valvuloplasty and regime for Extensively drug-resistant tuberculosis.

Keywords: XDR-TB, Hemoptysis, Bronchial artery embolization, Tetralogy of Fallot, ATT.

Copyright (C) 2020 The Author(s): This is an open-access article distributed under the terms of the Creative Commons Attribution 4.0 International License (CC BY-NC 4.0) which permits unrestricted use, distribution, and reproduction in any medium for non-commercial use provided the original author and source are credited.

\section{INTRODUCTION}

Incidence of the pulmonary tuberculosis is high in children with congenital heart disease, stated to be around 2.5 times more common than in normal children [1]. such cases are usually found in high flow acyanotic congenital heart disease [2]. However, there are only few case reports in low flow lesions like Tetralogy of Fallot [3].

We report a rare association of Tetralogy of Fallot with XDR-TB, not mentioned so far in the literature. The patient was successfully managed by bronchial artery embolization, venesections, intracardiac repair, valvuloplasty and appropriate antitubercular drugs.

\section{CASE REPORT}

A 22-year male, a non-smoker, presented in the Apollo hospitals New Delhi in 2013 with two month's history of loss of weight and appetite, intermittent fever for 15 days and recently two episodes of mild hemoptysis. On physical examination, he was found to be thin built having BMI of $16 \mathrm{~kg} / \mathrm{m}^{2}$. There was a grade-III digital clubbing and central cyanosis.
On auscultation there was a pansystolic murmur in $2^{\text {nd }}$ left intercostal space and diffuse continuous murmur over the left chest wall. There were crepitations bilaterally in the infrascapular area. The patient had previously been operated for Tetralogy of Fallot in the year 1997 when a Blalock Taussig shunt was performed. He was also being treated as a case of Isoniazid resistant pulmonary tuberculosis for a year before the presentation.

The patient's routine blood investigations revealed polycythemia ( $\mathrm{Hb} 20 \mathrm{gm} \%$ ) and type-II respiratory failure. The sputum stain for acid-fast bacilli was negative. His chest X-ray (Fig-1) and HRCT findings (Fig-2) were consistent with reduced left lung volume and fibrocavitatory changes. The echocardiography revealed a moderate-sized $[6 \times 5 \mathrm{~mm}]$ perimembranous Ventricular Septal Defect (VSD) with right infundibular and valvular pulmonary stenosis, aortic override of around 40\%, confluent branch pulmonary arteries and severe right ventricular hypertrophy. The echo findings were confirmed by CT pulmonary and cardiac angiography. 


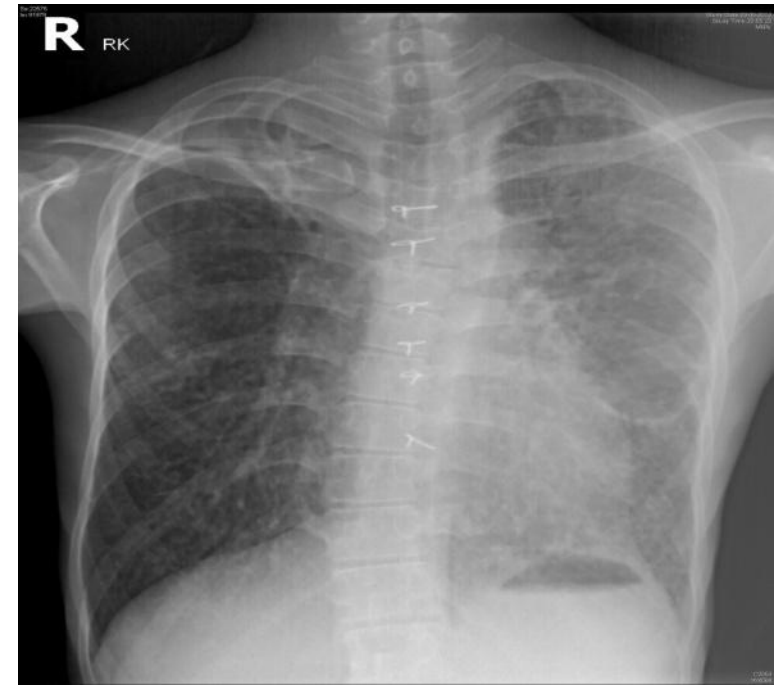

Fig-1: Chest $x-r a y$ showing bilateral fibrocavitation and volume loss

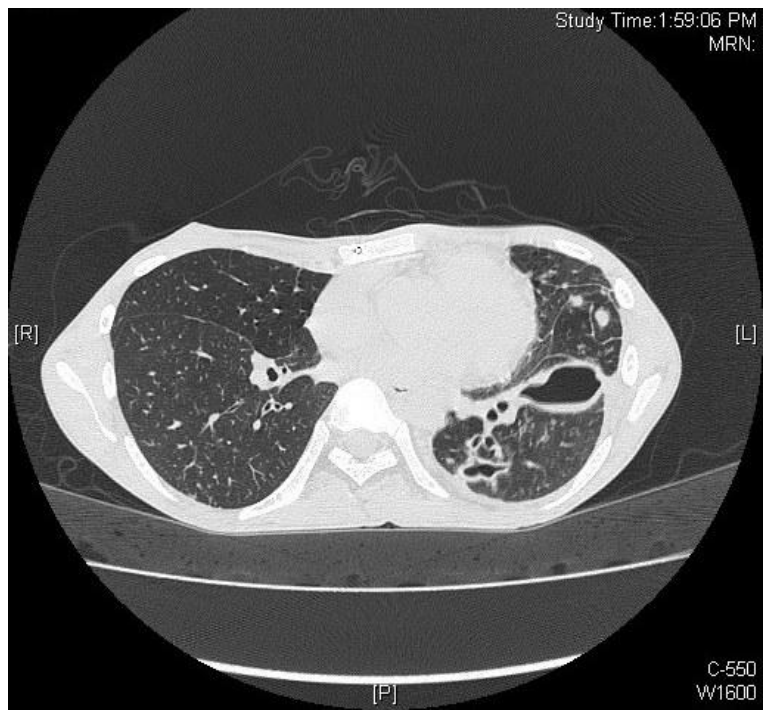

Fig-2: CAT scan showing nodular shadows and cavities

The patient underwent bronchoscopy for moderate hemoptysis. During bronchoscopy, there was a torrential bleed from the left side of the lung for which he was intubated and mechanically ventilated. The bronchoalveolar lavage (BAL) was negative for AFB Stain, and cultures showed growth of Klebsiella species. However, lavage was positive for Mycobacterium tuberculosis (MTB) DNA in the polymerase chain reaction (PCR). A bronchial artery angiography was performed, which showed multiple aortopulmonary collateral arteries (MAPCA) from the left internal mammary artery (LIMA) to bronchial arteries (Fig-3) for which embolization with metallic coiling (Fig-4) was done to stop the hemoptysis.

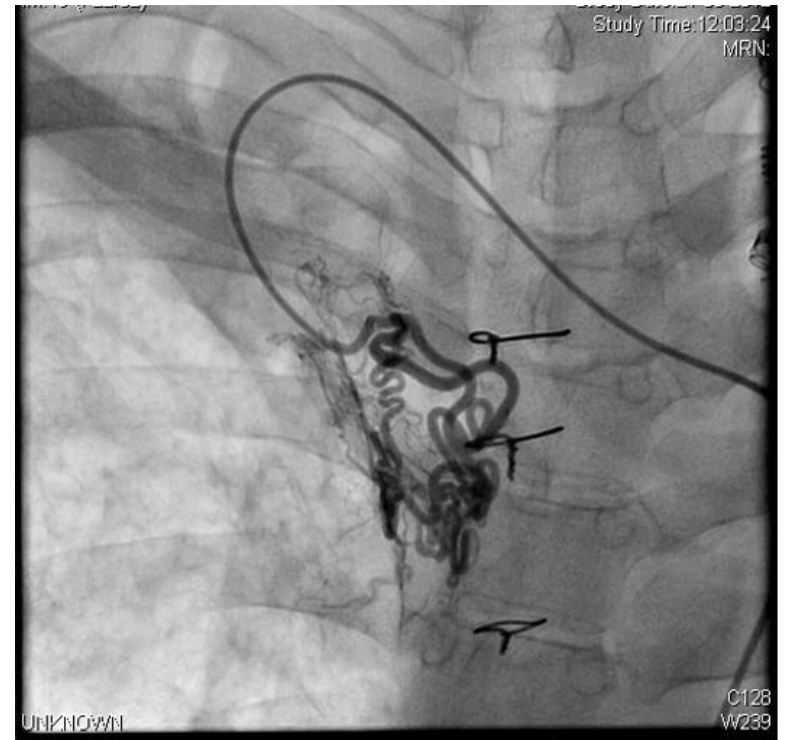

Fig-3: Bronchial artery angiography showing multiple collaterals

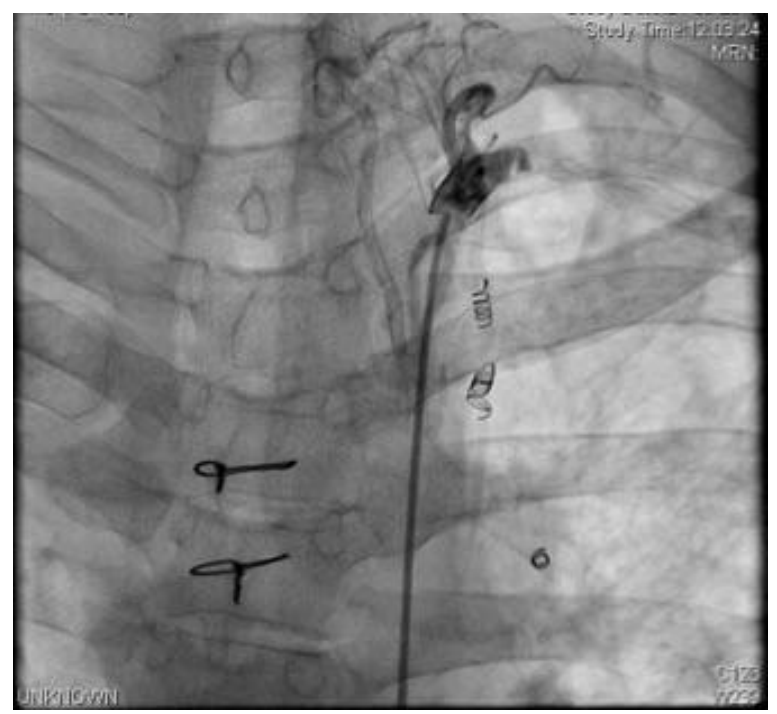

Fig-4: Coiling of the multiple vessels

The patient was extubated after 3 days and subsequently discharged on CAT-II RNTCP regimen. $\mathrm{He}$ has also advised venesection whenever his haemoglobin levels exceeded $18 \mathrm{gm} \%$. Two months later, he was readmitted with three episodes of haemoptysis, and bronchial artery embolization was done again for multiple collaterals. By this time, his AFB culture and sensitivity report were available, which showed resistance pattern to all $1^{\text {st }}$ line drugs and resistance to capreomycin, kanamycin and levofloxacin. The anti-TB regimen was switched to programmed RNTCP regimen for XDR TB and started on PAS, Clofazimine, Linezolid, Cycloserine, Amoxyclav and Moxifloxacin. Six months later he was again admitted with increased breathlessness at rest and severe hypoxia, as oxygen saturations were $60 \%$ on room air. The 2D-ECHO showed an increased gradient (80 $\mathrm{mmHg}$ ) in the pulmonary outflow tract. Cardiac catheterization and balloon pulmonary valvuloplasty was done. Post-procedure, his oxygen saturations were improved to $90 \%$ on room air, and 2D-ECHO showed a 
significant reduction in the flow gradient of 30 to 35 $\mathrm{mmHg}$. After eight months of XDR TB therapy, his two consecutive sputum AFB cultures taken one month apart found negative; hence he was continued on the same medications for the next 18 months. His sputum AFB culture was followed quarterly, and when three consecutive cultures during $\mathrm{CP}$ and at the end of treatment found negative, treatment was stopped after 26 months of a total duration of therapy.

On follow up, we observed an increase in appetite and weight gain of around $6 \mathrm{kgs}$ (from $39 \mathrm{kgs}$ to $45 \mathrm{kgs}$ ) with an increase in exercise tolerance and resolution of the polycythemia. There was also a dramatic improvement in his response to the anti-TB treatment with sputum conversion and satisfactory clearance of the lung lesions (Fig-5).

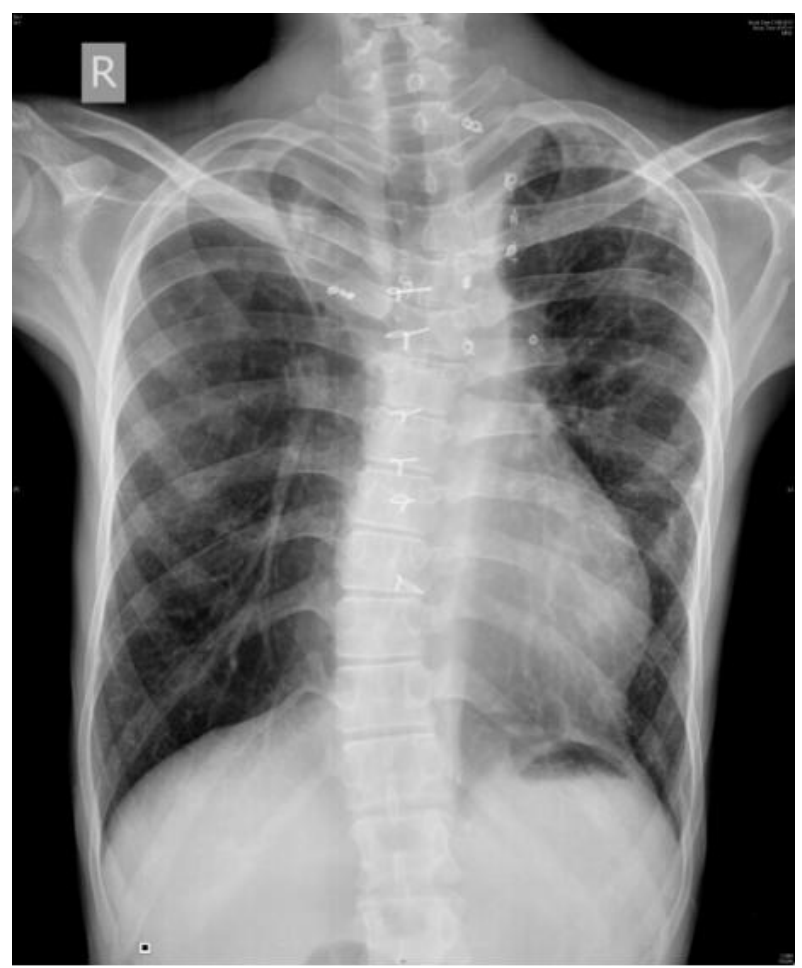

Fig-5: Chest $x$-ray after treatment showed reasonable clearance of left side shadows and cavitation

\section{DISCUSSION}

The Tetralogy of Fallot is serious congenital heart disease, presenting with the tetrad of obstruction of right ventricular outflow due to pulmonary artery atresia or stenosis, ventricular septal defect, right ventricular hypertrophy and the aorta straddling the ventricular septal defect [4].

The children affected with TOF are prone to hypoxic spells or blue spells during exertion or anxiety, due to right ventricular outflow tract stenosis (RVOT) and VSD [4, 5]. Patients frequently present with dyspnea, cyanosis and clubbing. Cyanosis is prominent in fingernails, toenails and mucous membranes. Growth and development of the child are retarded. In an attempt to compensate for low arterial oxygen concentration, the body responds with increased production of red blood cells or polycythemia. Red blood cell precursors may replace platelet stem cells in the bone marrow, leading to a thrombocytopenia, bleeding tendency and iron deficiency.

The aetiology and pathology of this disorder are not completely understood. It is postulated that these children were born with deficient cartilage in the tracheobronchial tree. The highly compliant bronchi collapse during coughing. This leads to poor drainage resulting in recurrent infections. The combination of TOF (low flow pulmonary lesion) with XDR TB, however, is not mentioned in the literature.

The oligaemic, hypoxic lungs are a suitable milieu for MTB to flourish. As in our case, once the blood flow improved in the pulmonary vasculature, the tubercular lung lesions responded satisfactorily to treatment. It was also a challenge to control haemoptysis in this patient where bronchial artery embolization was performed in two separate sittings. We also found that haemoptysis was less as long as haemoglobin was kept below $18 \mathrm{gm} \%$. Hence we performed periodic venesections.

TB is the leading infectious cause of death and one of the top 10 causes of death overall worldwide. In 2019, TB caused 1.4 million deaths, including 208000 among HIV-positive people [6].

Extensively drug-resistant tuberculosis or XDR-TB is defined as TB that has developed resistance to at least rifampicin and isoniazid as well as to any member of the quinolone family and at least one of the following second-line anti-TB injectable drugs: kanamycin, capreomycin, or amikacin [7, 8]. Our patient fits into this definition. The factors leading to the development of XDR-TB are probably not different from the usual causes of drug-resistant $\mathrm{TB}$, such as default treatment, non-compliance with guidelines, nonavailability of certain drugs, poor quality and poor storage condition of the drugs, inappropriate dose or combination, adverse reactions and malabsorption of the drugs.

The exact burden of XDR-TB is unknown as many countries lack infrastructure for case finding and diagnosis. By the end of 2017, 127 WHO Member States reported a total of 10,800 cases of XDR-TB, and $8.5 \%$ of cases of MDR-TB in 2017 were estimated to have been XDR-TB [9].

The treatment of XDR TB is difficult as well as a low success rate because of the limited number of available drugs and intolerance of the medications. The PMDT in India recommends 6-12 months of the intensive phase of Moxifloxacin, Capreomycin, Ethionamide, Cycloserine, Pyrazinamide, Linezolid, Clofazimine and Ethambutol, and 18 months 
continuation phase of Moxifloxacin, Ethionamide, Cycloserine, Linezolid, Clofazimine and Ethambutol [10]. In case of resistance to both Ethambutol and pyrazinamide, PAS can be added to the intensive and continuation phase. In case of resistance to all secondline injectables (SLIs) replace them with PAS, Clofazimine and Linezolid.

The bedaquiline and delamanid were approved by WHO for use in the treatment of adults with drugresistant TB in 2013 and 2014 respectively [11-13]. The two medicines have now been integrated into the algorithms for the design of regimens for multidrugresistant TB and extensively drug-resistant TB patients [14].

Recently a three-drug regimen consisting of bedaquiline, pretomanid and linezolid- collectively referred to as the BPaL regimen approved by the FDA for use as a short-term therapy in highly resistant tuberculosis [15]. The success of the XDR TB treatment highly depends upon the adherence and completion of the treatment course. A study in the Tomsk oblast of Russia reported that 14 out of 29 (48.3\%) patients with XDR-TB successfully completed treatment [16].

\section{CONCLUSION}

In conclusion, the association of TOF with XDR-TB is so far not mentioned in the literature. Surgical treatment for Tetralogy of Fallot and drug sensitivity based Anti-tubercular Drugs is the ideal protocol that should be followed. Preservation of as much lung parenchyma as possible by preventing the tuberculosis spread is important; this may be possible by the improvement of pulmonary blood flow.

\section{ACKNOWLEDGEMENT}

The authors acknowledged the contribution received from the microbiology department of Apollo Hospitals, New Delhi.

\section{REFERENCES}

1. Van der Merwe, P. L., Kalis, N., Schaaf, H. S., Nel, E. H., \& Gie, R. P. (1995). Risk of pulmonary tuberculosis in children with congenital heart disease. Pediatric cardiology, 16(4), 172-175.

2. Radović, M., Ristić, L., Stanković, I., \& Petrović, D. (2010). Rare congenital hearth disease as a cause of tuberculosis activation. Medicinski pregled, 63(7-8), 565-569.

3. Günay, E., Günay, S., Karakuş, G., Şahin, T., Görgün, D., Tursun, I., \& Dural, C. (2012). Pulmonary tuberculosis in an adult patient with tetralogy of Fallot. Hippokratia, 16(3), 286.

4. James, R. Z. (2001). Tetralogy of Fallot Moss and Adams heart disease in infants, children and adolescents. $6^{\text {th }}$ ed. Hippincott William Wilkins Company: 880-902.

5. Daniel Bernstein tetralogy of fallot. Nelson textbook of pediatrics $16^{\text {th }}$ ed. W.B. Sanders company:1389-1389 2000.

6. Sustainable development goals [website]. New York: United Nations; (https://sustainabledevelopment.un.org/topics/sust ainabledevelopmentgoals, accessed 20 July 2020).

7. Centers for Disease Control and Prevention (CDC). (2006). Emergence of Mycobacterium tuberculosis with extensive resistance to secondline drugs - worldwide 2000-2004. MMWR Morb Mortal Wkly Rep. 55:301-5.

8. Centers for Disease Control and Prevention (CDC). (2006). Notice to readers: Revised definition of extensively drug-resistant tuberculosis. MMWR Morb Mortal Wkly Rep. 55:1176.

9. "Global tuberculosis report". World Health Organization (WHO). 28 February 2019. Retrieved 18 August 2019.

10. Guidelines on the Programmatic Management of Drug-Resistant TB (PMDT) in India: Central TB Division, Directorate General of Health Services, Ministry of Health \& Family Welfare, Government of India, New Delhi, 2017.

11. The use of bedaquiline in the treatment of multidrug resistant tuberculosis - Interim policy guidance," World Health Organization, Geneva, 2013.

12. The use of delamanid in the treatment of multidrug-resistant tuberculosis. Interim policy guidance (WHO/HTM/TB/2014.23) [Internet]. Geneva, World Health Organization. 2014.

13. The use of delamanid in the treatment of multidrug-resistant tuberculosis - Interim policy guidance," New Delhi, 2014.

14. Companion handbook to the WHO guidelines for the programmatic management of drug-resistant tuberculosis. (WHO/HTM/TB/2014.11) [Internet]. Geneva, World Health Organization. 2015.Availablefrom: http://apps.who.int/iris/bitstream/10665/130918/1/ 9789241548809_eng.pdf

15. TB Alliance. Pretomanid and BPaL Regimen for Treatment of Highly Resistant Tuberculosis. Oral presentation at: Antimicrobial Drugs Advisory Committee; June 6, 2019; Silver Spring, MD.

16. Keshavjee, S., Gelmanova, I. Y., Farmer, P. E., Mishustin, S. P., Strelis, A. K., Andreev, Y. G., ... \& Furin, J. J. (2008). Treatment of extensively drug-resistant tuberculosis in Tomsk, Russia: a retrospective cohort study. The Lancet, 372(9647), 1403-1409. 